\title{
BIG DATA COMO FONTE DE INOVAÇÃO EM MUSEUS: o estudo de caso do Museu Britânico
}

\author{
BIG DATA AS INNOVATION SOURCE IN MUSEUMS: \\ the case study of The British Museum
}

Fernanda Miranda de Vasconcellos Motta ${ }^{1}$

Cátia Rodrigues Barbosa²

Ricardo Rodrigues Barbosa ${ }^{3}$

\begin{abstract}
RESUMO
No cenário contemporâneo, os museus têm seus processos influenciados pelas transformações digitais. Eles são centros informacionais que têm o desafio de coletar, organizar, processar, distribuir e analisar um volume exponencial de dados, em formatos diversos, obtidos de diferentes fontes. A utilização do Big Data em museus possibilita a gestão e análise de dados complexos, gerando informações estratégicas que dão embasamento à gestão institucional. A partir dessa perspectiva, tem-se como propósito pesquisar, através do método de estudo de caso, os processos de gestão e análise de dados do Museu Britânico. Este é o segundo museu mais visitado do mundo e tem como diretriz estratégica ser um museu para 0 cidadão global, amplamente acessível. É possível observar que o uso de Big Data, no caso estudado, é fonte de inovação, estimulando o desenvolvimento dos projetos ResearchSpace - espaço colaborativo de criação, gestão e compartilhamento de conhecimento; e The Museum of The World - plataforma virtual interativa.
\end{abstract}

Palavras-chave: Transformações Digitais. Big Data. Inovação. Museus. Museu Britânico.

\begin{abstract}
In the contemporary scenario, museums have their processes influenced by digital transformations. They are information centers that have the challenge of collecting, organizing, processing, distributing and analyzing an exponential volume of data, in different formats, obtained from different sources. The use of Big Data in museums enables the management and analysis of complex data, generating strategic information that supports institutional management. In this perspective, the article aims to investigate, through the method of case study, the data management and analysis processes of The British Museum. This is the second most visited museum in the world and its strategic guideline is a museum for the global citizen, widely accessible. It is possible to observe that the use of Big Data in the studied case is a source of innovation, stimulating the development of projects ResearchSpace - a collaborative space for creation, management and knowledge sharing; and The Museum of The World - an interactive virtual platform.
\end{abstract}

Keywords: Digital Transformations. Big Data. Innovation. Museums. The British Museum.

Artigo recebido em 16/01/2019 e aceito para publicação em 16/03/2019

1 Mestre em Administração pela Universidade Federal de Minas Gerais, Brasil. Graduação em Comunicação Social pela Pontifícia Universidade Católica de Minas Gerais, Brasil. Doutoranda no Programa de Pós-graduação em Gestão e Organização do Conhecimento da Universidade Federal de Minas Gerais, Brasil. E-mail: fernandavasc@gmail.com.

2 Doutorado em Muséologie Des Sciences Naturelles Et Humaines pelo Ecole Du Muséum National D’histoire Naturelle de Paris, França. Professora titular do Departamento de Organização e Tratamento da Informação da Escola de Ciência da Informação da Universidade Federal de Minas Gerais, Brasil. Docente permanente do Programa de Pós-graduação em Gestão e Organização do Conhecimento da Universidade Federal de Minas Gerais, Brasil. E-mail: catiarb@eci.ufmg.br.

3 Doutorado em Administração de Empresas pela Columbia University, Estados Unidos. Professor titular do Departamento de Organização e Tratamento da Informação da Escola de Ciência da Informação da Universidade Federal de Minas Gerais, Brasil. Docente permanente do Programa de Pós-graduação em Gestão e Organização do Conhecimento da Universidade Federal de Minas Gerais, Brasil. E-mail: ricardobarbosa@ufmg.br. 


\section{INTRODUÇÃ $0^{4}$}

No cenário contemporâneo, é fundamental que as organizações saibam criar, organizar, processar e disseminar informações, para gerar novos conhecimentos, planejar produtos e serviços e melhorar processos. Essa dinâmica, mediada por recursos tecnológicos avançados, fornece suporte ao processo de tomada de decisões estratégicas. "0 que emerge é um conjunto de significados compartilhados e modelos mentais que a organização utiliza para planejar e tomar decisões" (CHO0, 2003, p.51).

Os sistemas de gestão da informação vêm tornando-se mais complexos, processando dados extraídos de fontes diversas, tais como redes wi-fi, redes sociais, bibliotecas digitais, coleções multimídia, aplicativos, bancos de dados e outras fontes. Esse crescimento exponencial de dados requer não apenas investimento em tecnologias de processamento, mas também em ferramentas de análise e visualização dos dados, com o propósito de transformá-los em informações estratégicas. Nesse contexto, emerge o Big Data - que se refere à gestão de complexos conjuntos de dados, com o intuito de evidenciar, por meio de processos analíticos associados, tendências e conexões com potencial de contribuir para a estratégia de negócios (GANDOMI; HAIDER, 2015).

Museus, como centros informacionais, têm seus processos reconfigurados pelas transformações digitais (BAUTISTA, 2014). Eles coletam volumes cada vez maiores de dados e um desafio de gestão é disponibilizar informações relevantes para as pessoas certas, na linguagem adequada, em tempo oportuno (PERERA; CHANDRA, 2011). 0 Big Data torna-se um recurso aplicável a tais instituições, alinhando-se com seu direcionamento estratégico.

A partir dessas considerações iniciais, foram delineadas as seguintes questões de pesquisa que norteiam este estudo:

- Quais são os processos relacionados à gestão de Big Data?

- Como os processos de Big Data se integram à gestão dos museus?

- $\quad$ Os processos de Big Data são fontes de inovação para os museus?

Com o intuito de investigar estas questões, parte-se da análise do fenômeno Big Data, no contexto da revolução digital. Em seguida, busca-se entender como os museus vêm reconfigurando seus processos, com base nas transformações digitais. É abordado, então, o estudo de caso do Museu Britânico, com o intuito de pesquisar como os processos de gestão e análise de Big Data dão suporte à gestão e podem ser fonte de inovação.

40 desenvolvimento deste artigo contou com o apoio da Fundação de Amparo à Pesquisa do Estado de Minas Gerais - Fapemig. 


\section{METODOLOGIA}

A abordagem metodológica adotada neste artigo é qualitativa, com finalidades exploratórias e descritivas. Inicialmente, são levantadas bases teóricas para melhor compreender o fenômeno em estudo. Na etapa seguinte, busca-se descrevê-lo e interpretá-lo à luz das teorias. Essa abordagem considera 0 ambiente como fonte direta de dados, sendo que os pesquisadores têm papel-chave na pesquisa. 0 foco está nos processos em estudo e em sua análise (FREITAS; JABBOUR, 2011).

0 método adotado é o estudo de caso, que consiste em reunir informações sistemáticas sobre o fenômeno, possibilitando a compreensão do contexto no qual ele se manifesta e das variáveis com as quais ele se relaciona (PATTON, 2002). Esse método é indicado quando os pesquisadores buscam conhecer, de forma mais aprofundada, os fenômenos da vida contemporânea, analisando casos reais, guiados questões de pesquisa. 0 estudo de caso é delimitado e descrito com base em certos parâmetros, englobando tanto o histórico institucional quanto 0 detalhamento de processos em análise, com 0 objetivo de favorecer o entendimento do fenômeno, em toda sua complexidade. 0 protocolo do estudo de caso envolve a definição de questões de pesquisa, o desenvolvimento de temas para embasamento teórico, a escolha das fontes de coleta de dados e dos procedimentos de análise das informações. Nesse tipo de estudo, destaca-se a importância dos pesquisadores zelarem pela confiabilidade dos dados, sendo criteriosos na escolha das fontes (CRESWELL, 2013). Os procedimentos de coleta de dados adotados baseiam-se em pesquisas bibliográficas, documentais e de etnografia digital, sendo esta última uma das metodologias apropriadas ao estudo empírico de fenômenos da internet (FRAGOSO et al., 2013). Sua adoção permite analisar como as tecnologias da internet estão sendo empregadas em determinado contexto, gerando informações para o desenvolvimento do estudo de caso.

\section{A REVOLUÇÃO DIGITAL E 0 FENÔMENO BIG DATA}

Com a revolução digital, inovações tecnológicas geram um crescimento exponencial de dados e impulsionam avanços na gestão estratégica da informação (KEENE, 2010). As tecnologias se tornam mais integradas, a capacidade analítica de dados se amplia, bem como as possibilidades de desenvolvimento de projetos customizados. 0 escopo dessa revolução não se restringe a máquinas e a sistemas conectados e inteligentes. A fusão das tecnologias e sua interação, através de domínios físicos e digitais, são os pilares a diferenciam das demais (SCHWAB, 2016). Novas regras de competitividade em negócios são adotadas e a ênfase está em agregar valor a produtos e serviços. Estabelece-se a 
possibilidade de desenvolver ecossistemas inovadores, que integrem as operações organizacionais e as conectem ao ambiente externo. A lógica que sustenta o crescimento global envolve a mobilização de diferentes formas de inteligência, a integração sistêmica das operações, o compartilhamento de informações, a abertura à aprendizagem e o espírito de colaboração. Nesse contexto, Big Data representa uma estratégia de gestão informacional que influencia o ecossistema organizacional, transforma processos gerenciais e estimula a inovação. Ele envolve a adoção de sistemas tecnológicos avançados e o desenvolvimento de habilidades intelectuais de alto nível para coletar, estocar, organizar, extrair, analisar e distribuir dados (GANDOMI; HAIDER, 2015). A gestão e análise apropriadas dos dados podem gerar novos conhecimentos e favorecer a agilidade de resposta da organização aos desafios e oportunidades que se apresentam (CHEN; ZHANG, 2014).

Wamba et al. (2015) esclarecem que o fenômeno Big Data é derivado da ampla difusão e adoção de plataformas virtuais, mídias móveis, redes sociais e de conceitos relacionados à Internet das Coisas. Os referidos autores (2015, p. 235) mencionam a esse respeito: "nós definimos Big Data como uma abordagem holística para gerenciar, processar e analisar cinco Vs, de forma a criar insights acionáveis para a entrega sustentável de valor". No quadro apresentado a seguir estão descritas as dimensões relacionadas ao fenômeno.

Quadro 1 - Dimensões do Fenômeno Big Data

\begin{tabular}{|c|c|}
\hline Volume & $\begin{array}{c}\text { Magnitude dos dados. Grande número de dados } \\
\text { estocados ou entrada de grande número de registros no } \\
\text { sistema. }\end{array}$ \\
\hline Velocidade & $\begin{array}{c}\text { Frequência ou velocidade de geração e entrega dos } \\
\text { dados. Capacidade de processamento em tempo real. }\end{array}$ \\
\hline Variedade & $\begin{array}{r}\text { Integração dos dados. Eles são gerados a partir de } \\
\text { diversas fontes e em diferentes formatos. }\end{array}$ \\
\hline Valor & $\begin{array}{r}\text { Importância de se extrair benefícios institucionais e } \\
\text { econômicos da análise dos dados. }\end{array}$ \\
\hline Veracidade & $\begin{array}{c}\text { Relevância da qualidade dos dados, em termos de } \\
\text { confiabilidade das fontes e dos dados delas extraídos. }\end{array}$ \\
\hline
\end{tabular}

Fonte: adaptado de Wamba et al. (2015) 
De forma complementar, Jin et al. (2015) argumentam que, em uma perspectiva macro, Big Data pode ser entendido como um elo que conecta o mundo físico ao ciberespaço. 0 fenômeno associa-se à Internet das Coisas na medida em que esta representa uma complexa infraestrutura que habilita serviços avançados, por meio de interconexões físicas e virtuais de objetos com redes. Dispositivos com tecnologia digital embarcada transmitem e recebem dados o tempo todo, sendo que Big Data e computação em nuvem são estruturas tecnológicas subjacentes. De acordo com Hammoudi et al. (2018), ambientes físicos e virtuais inteligentes reagem a estímulos, identificam demandas, interagem com pessoas, tomam decisões. A Internet das Coisas é parte expressiva do fenômeno de crescimento exponencial de dados, sendo estes gerados por uma miríade de dispositivos que fornecem informações sobre usuários e contextos de uso.

Gandomi e Haider (2015) argumentam que a maioria dos dados que compõem o Big Data são semi-estruturados ou não-estruturados, sendo que os dados estruturados, codificados em linguagem computacional, representam a menor parte. Os referidos autores (2015) esclarecem que dados nãoestruturados consistem em imagens, vídeos, áudios, textos de redes sociais e de páginas da internet, em linguagem natural. Os dados semi-estruturados, por sua vez, são os que adotam linguagem textual para troca de dados na web, com tags definidas pelos usuários que tornam possível sua leitura por máquinas.

Um alto nível de variedade, característica distintiva do Big Data, não é necessariamente novo. Organizações têm obtido dados não-estruturados de fontes internas (exemplo: sensores) e de fontes externas (exemplo: mídias sociais). Contudo, a emergência de novas tecnologias de gestão e análise de dados, que habilitam as organizações a aplicar dados em seus processos de negócios, é 0 aspecto inovador (GANDOMI; HAIDER, 2015, p. 138).

0 processo de Big Data tem início, segundo Daish (2017), com a entrada de dados de diversas fontes, que são integrados e transformados, por meio de sistemas avançados de hardware e software. Os conjuntos de dados resultantes são armazenados em nuvem. Em seguida, eles são analisados, por meio de ferramentas que permitem estabelecer relações, tendências e padrões de comportamento. Essas análises possibilitam gerar ideias que dão suporte à gestão institucional. A figura apresentada a seguir ilustra o processo. 
Figura 1 - Processos de gestão e análise de Big Data

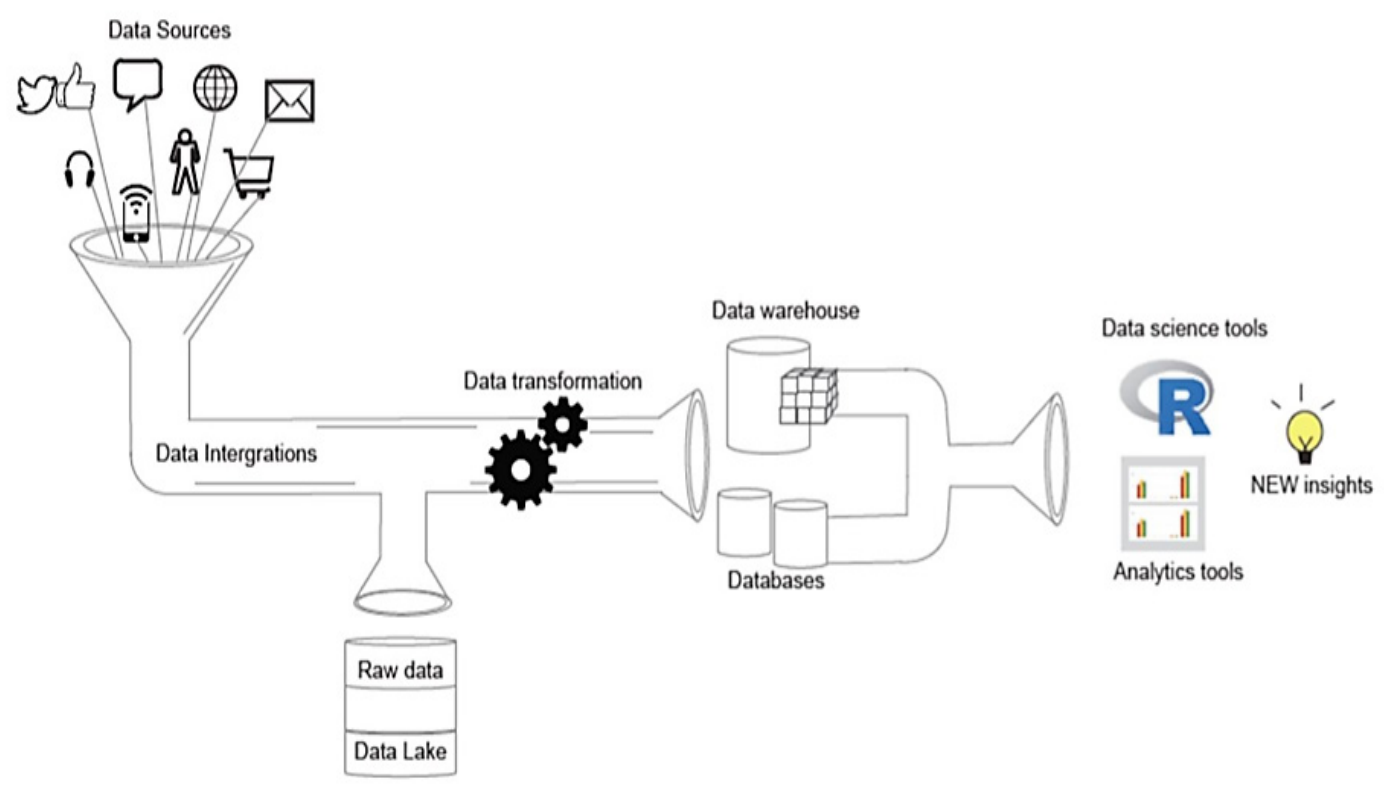

Fonte: Daish (2017)

Dados sobre localização geoespacial, navegação na internet, características demográficas e psicográficas de usuários, padrões de uso e consumo de produtos e serviços, comentários e avaliações, podem ser analisados e empregados em decisões de negócios para melhorar processos, criar valor para os públicos-alvo e gerar receitas. 0 investimento nessas inovações tecnológicas possibilita que as organizações criem inteligência, em tempo real, a partir de altos volumes de dados (CHEN; ZHANG, 2014).

Enquanto que a gestão envolve processos e tecnologias para agrupar, processar e estocar dados, a análise refere-se ao uso de técnicas para extrair inteligência deles. Ela cria sentido para os dados, estabelecendo conexões e embasando soluções criativas de visualização e compartilhamento de informações. Procedimentos de análise, de acordo com Gandomi e Hainder (2015), podem ser aplicados a arquivos de textos, de multimídia (imagem, áudio, vídeo) e das redes sociais. Eles envolvem tratamentos matemáticos, estatísticos, linguística computacional e aprendizagem de máquina.

Uma estratégia analítica associada ao Big Data é a web semântica, que, de acordo com Bax (2012), é usada para integração de dados em larga escala, a partir de fontes de dados autônomas e heterogêneas. São usados Uniform Resource Identifier - URIS - para identificar recursos informacionais e objetos, por meio de um modelo de dados extensível denominado Resource Description Framework$R D F$. Esse modelo possibilita que referência (o objeto em si) e sentido (descrição do objeto) associem- 
se. A web semântica é aplicável ao ambiente digital em que pessoas e máquinas interagem, de forma colaborativa, servindo para mapear não só recursos informacionais, mas todas as coisas do mundo, além de ideias e conceitos a elas relacionados. Bax (2012, p. 91) argumenta que "[...] a web semântica pode ser vista como uma forma de linguagem compartilhada entre múltiplos agentes, homens e máquinas. 0 significado de uma URI equivale ao uso comunitário que os agentes fazem dessa URI”. Cria-se uma linguagem social e colaborativa capaz de enriquecer os dados processados.

Outras técnicas são análise de sentimento - que mapeia as opiniões das pessoas sobre empresas, produtos, serviços, processos; detecção de comunidades virtuais e de influências sociais; e predição de links. Análises preditivas são aplicadas para identificar padrões e relacionamentos entre históricos de dados passados e correntes, embasando decisões futuras (GANDOMI; HAINDER, 2015). A análise de Big Data, de acordo com Chen e Zhang (2014), cria possibilidades mais intuitivas e funcionais de representação do conhecimento, explorando múltiplas dimensões como estética, usabilidade e interação, para aprimorar a experiência dos usuários.

\subsection{Transformações digitais em museus}

No início deste século, as Tecnologias de Informação e Comunicação - TICs - passaram a estimular, com mais intensidade, transformações digitais nos espaços museais, na gestão de seus acervos e na relação com seus públicos. Keene (2011, p. 1) comenta a esse respeito: "nós costumávamos construir coleções de objetos. Agora podemos criar coleções de informações também”. Com as TICs, torna-se possível capturar, de forma exponencial, a dimensão informacional das coleções. Deloche (2001) observa que a apresentação do objeto museal passa a ser autônoma, não mais se limitando às fronteiras físicas da instituição museu. Inaugura-se o fenômeno do "museu expandido", em que os campos físico e virtual se complementam, estimulando fluxos informacionais diversos. Nesse sentido, Gobira (2018) argumenta que a ideia de museu já não se liga tanto às instalações físicas ou aos objetos em exposição, mas sim às relações entre os sujeitos e os ambientes museais físicos ou virtuais. "A computação trouxe novas possibilidades para a sociedade, inclusive na arte e na cultura" (GOBIRA, 2018, p. 85).

0 advento das TICs viabilizou a digitalização de acervos, a criação ambientes virtuais, 0 desenvolvimento de conteúdos interativos, o crescimento da comunicação móvel, as experiências imersivas, dentre outras inovações. Esse fenômeno se vincula ao conceito de museu participatório, 
englobando comunicação multidirecional, livre circulação de informações e colaboração entre diferentes agentes museais (SIMON, 2010). Estratégias de crowdsourcing passam a ser planejadas pelos gestores dos museus. Elas envolvem interações sociais entre diversos agentes, com a mediação da internet e das mídias digitais, para o desenvolvimento de projetos, ideias, serviços e conteúdos (MARTINS, 2017).

Para fornecer o adequado suporte à cultura participatória que emerge em museus, a gestão estratégica da informação se aprimora, impulsionada pelas inovações tecnológicas. "Museus são para Iongo prazo: uma importante parte do futuro de longo prazo reside na informação" (KEENE, 2011, P. 8). Em termos de comunicação com os públicos, Keene (2011) argumenta que a internet abre oportunidades de broadcasting e narrowcasting para os museus. Broadcasting, conforme esclarece a autora (2011), relaciona-se com tecnologias e processos que possibilitam que as informações cheguem a qualquer pessoa, em qualquer lugar do mundo. Já narrowcasting envolve o direcionamento de recursos para 0 atendimento às demandas de públicos específicos, entregando conteúdos personalizados. Uma base para o adequado planejamento de tais estratégias reside na criação de uma cultura institucional voltada para a gestão e análise de dados, nos museus.

\section{ESTUDO DE CASO DO MUSEU BRITÂNICO}

0 Museu Britânico, situado em Londres, foi o primeiro museu nacional do mundo e a primeira instituição pública britânica. Foi fundado em 1753, pelo parlamento inglês, com o propósito de representar um espaço dedicado ao estudo das sociedades humanas e de suas transformações, através do tempo e do espaço. Abriu-se à visitação pública em 1759, com acesso público e gratuito à coleção permanente. De acordo com informações extraídas de seu website, ele é o segundo museu mais visitado do mundo, com mais de seis milhões de visitantes físicos e mais de oito milhões de visitantes virtuais, por ano. A extensa coleção de objetos, com cerca de oito milhões de itens, abrange desde objetos arcaicos aos contemporâneos. A base de dados Collection Online, implantada em 2007, possibilita que os visitantes virtuais pesquisem mais de dois milhões de registros, associados a mais de quatro milhões de objetos e a mais de um milhão de imagens digitais. Na atualidade, 0 Museu Britânico é considerado um dos mais importantes centros de pesquisa a respeito da cultura material da humanidade, abarcando objetos que correspondem a cerca de dois milhões de anos da história da humanidade. 


\subsection{Análise estratégica}

Com base no relatório Towards 2020 - The British Museum Strategy, o museu tem como meta, até 2020, investir em estratégias que reforcem seu posicionamento de museu para o cidadão global. A ideia é que a coleção esteja cada vez mais acessível e explorável, em diversos pontos do mundo. A seguir são indicados alguns desafios e oportunidades de gestão.

Quadro 2 - Desafios e oportunidades para o Museu Britânico

$\checkmark$ A visitação online ao museu cresce, a cada ano, demandando estratégias inovadoras de engajamento e interatividade com o público virtual.

Há 0 desafio de estimular as visitações físicas à coleção permanente e assegurar que 0 acesso a ela continue sendo público e gratuito.

0 governo é o principal financiador do museu, mas vêm crescendo as receitas vindas de exposições temporárias, consultorias, treinamentos, publicações, comércio físico e eletrônico.

$\checkmark 0$ programa de relacionamento conta com cerca de 40.000 associados, tem alcance global, e há tendência de expansão.

$\checkmark$ A navegação nos espaços físicos e virtuais do museu está cada vez mais relacionada ao uso de tecnologias interativas, ao desenvolvimento de narrativas colaborativas e à customização da experiência. 0 uso de tecnologias móveis está em crescimento.

Há demanda crescente dos públicos por extensões de conteúdo - tais como palestras, debates e interações online que relacionem temas da atualidade com 0 acervo do museu.

$\checkmark$ A arquitetura tradicional do conhecimento vem sendo dissolvida. Aumenta a necessidade de se pensar em novas formas de comunicar o acervo e de engajar diferentes públicos-alvo em relação à experiência de museu.

A atuação em rede e o posicionamento do museu como centro de pesquisas global sobre a história da humanidade são prioridades estratégicas.

Fonte: adaptado do relatório Towards 2020 - The British Museum Srategy

Com base nesses aspectos, o referido relatório contempla algumas diretrizes estratégicas para a gestão do Museu Britânico, até 2020: 
- Estimular a visitação física à coleção permanente do museu, criando ambientes agradáveis e interativos de visitação, planejando conteúdos e roteiros de visitação customizados.

- $\quad$ Vincular o museu a questões contemporâneas que possam ser exploradas e discutidas, a partir do acervo, de acordo com os diferentes perfis dos públicos-alvo.

- $\quad$ Desenvolver parcerias estratégicas com instituições de educação, pesquisa e tecnologia, para reforçar o posicionamento do museu como fonte de conhecimento global.

- Investir em narrativas interativas sobre o acervo, valorizando diferentes vozes e perspectivas da história, para que a experiência de museu faça sentido para os públicos.

- $\quad$ Estender o alcance do museu, por meio da experiência virtual, tornando-a cada vez mais acessível, interativa e colaborativa.

- $\quad$ Fomentar a atuação em rede, de forma articulada com outras instituições e com os diversos agentes que se relacionam com o museu, investindo em estratégias de crowdsourcing.

- Considerar o museu como centro de referência global em pesquisas, articulando ambientes, pessoas e tecnologias capazes de criar a adequada infraestrutura para esse propósito.

0 investimento em inovações tecnológicas e a valorização da expertise dos profissionais do museu, por meio de processos bem planejados de pesquisa, organização, exposição e mediação, são bases para o alcance dos objetivos de desenvolvimento institucional estabelecidos.

\subsection{Big Data no Museu Britânico}

Com o propósito de tornar o acervo amplamente acessível, a gestão do museu vem tornando suas operações orientadas por dados. 0 desafio é auxiliar seu enorme e heterogêneo público a explorar o também enorme acervo, por meio de estratégias voltadas ao engajamento deste e ao aprimoramento da experiência de museu. A equipe de Big Data, composta por cientistas de dados, é responsável por prover soluções inovadoras de processamento, navegação e visualização de dados. Daish (2017) menciona que uma pesquisa conduzida pela equipe, em 2015, indicou mais de 250 fontes de dados diferentes, dentre elas bases de dados; website, páginas web, audioguias, aplicativos para mídias móveis, redes wi-fi e redes sociais tais como facebook, flickr, twitter, instagram, periscope. 
Figura 2 - Principais fontes de dados do Museu Britânico

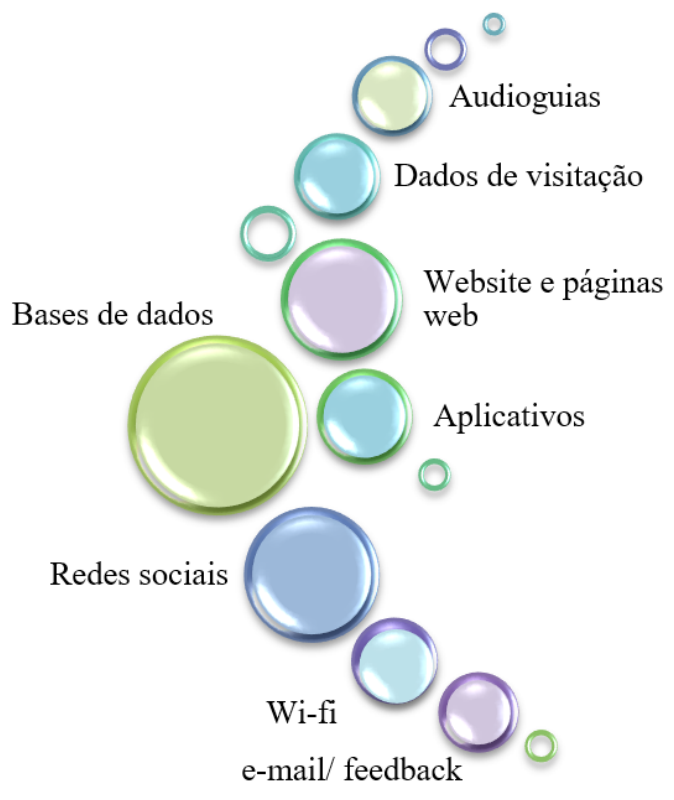

Fonte: adaptado de Daish (2017)

A partir de 2017, a equipe passou a investir na integração dos dados de diversas fontes, advindas de diferentes setores do museu, abarcando atividades de engajamento dos visitantes, comerciais, operacionais, financeiras, de recursos humanos, de documentação e conservação das coleções e de pesquisa científica.

Figura 3 - Dados agrupados por setores

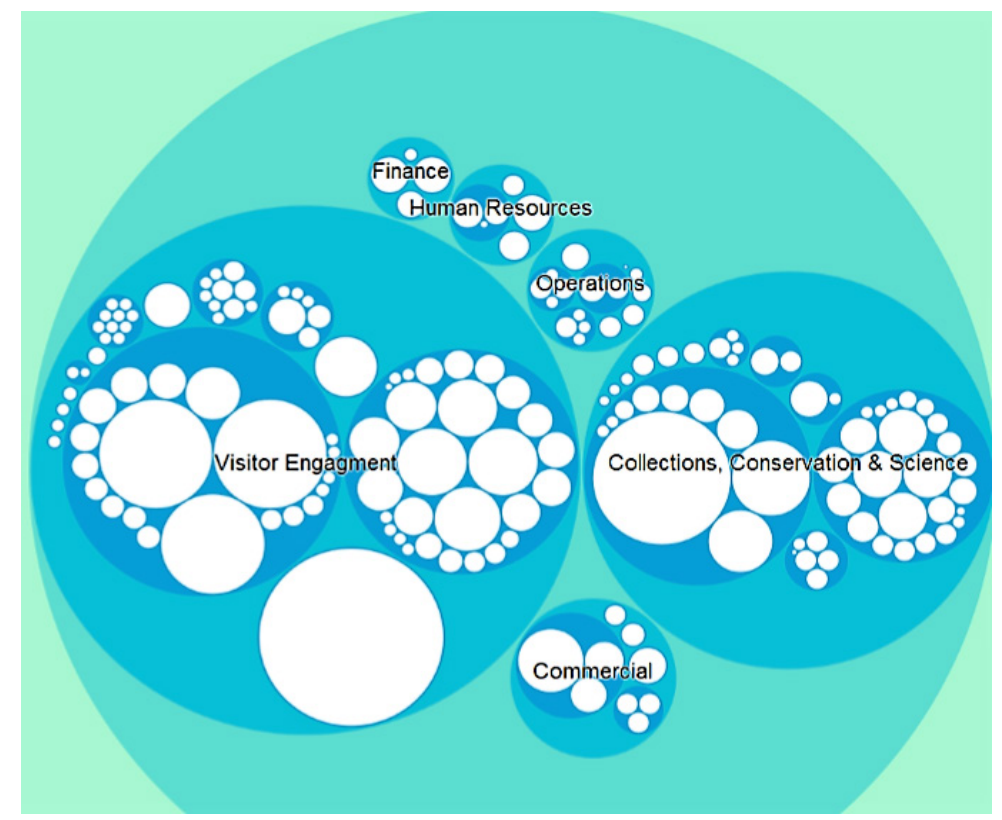

Fonte: Daish (2017) 
Todos os dados obtidos pela equipe do museu são reagrupados, processados e armazenados através do sistema Azure, da Microsoft (Azure SQL Server e Azure Blob Storage). Após esta etapa, são aplicadas ferramentas de análise e visualização de dados. Os softwares Azure Power BI e Azure Machine Learning Studio são usados nesse processo.

Com o intuito de alinhar os processos de gestão e análise de Big Data às demandas gerenciais, foram estabelecidas as seguintes etapas a serem cumpridas:

\section{Quadro 3 - Etapas do Big Data}

1. Identificar problemas ou questões que merecem análise.

2. Acordar com gestores e stakeholders quais as questões a serem pesquisadas.

3. Identificar os dados disponíveis.

4. Identificar como melhor integrar os dados.

5. Decidir como minerar, transformar e lidar com lacunas nos dados.

6. Definir como os dados serão gerenciados, estocados, acessados e visualizados.

7. Analisar dados.

8. Criar relatórios e interfaces para visualização.

9. Prover insights gerenciais e diretrizes para construção de narrativas multimídia.

10. Discutir como aplicar os resultados no processo de tomada de decisões.

11. Debater sobre como melhor coletar dados em situações futuras (novas formas de coleta, novos dados e melhorias nos métodos existentes).

12. Fomentar novas questões que embasem esforços de pesquisa e análise.

Fonte: adaptado de Daish (2017)

Daish (2017) menciona que não se trata apenas de implantar novas tecnologias, e sim de promover uma transformação estratégica maior. Ela envolve pensar em como os dados coletados de diversas fontes podem ser usados para ampliar a visibilidade e acesso aos acervos, aprimorar as experiências e 0 engajamento dos públicos, elevar a eficácia da gestão de recursos organizacionais e criar novas fontes de receita. 


\subsection{0 projeto ResearchSpace, baseado em web semântica}

0 Museu Britânico, em parceria com a Fundação Andrew W. Mellon, integrou o projeto ResearchSpace, desenvolvido pela fundação e baseado em web semântica, à sua base de dados Collection Online. Tem-se um ambiente virtual que funciona tanto como mecanismo de pesquisa do acervo do museu quanto como ambiente interativo, por meio da estrutura de dados abertos linkados. Essa dinâmica permite o reuso do acervo digital do museu e o engajamento de pesquisadores, acadêmicos e desenvolvedores em processos permanentes de construção, gestão e compartilhamento de conhecimento. Os usuários podem tanto acessar e editar dados quanto contribuir com a inserção de novos dados, na forma de anotações RDF, a partir de um modelo de representação gráfica das informações.

Figura 4 - Ambiente colaborativo ResearchSpace

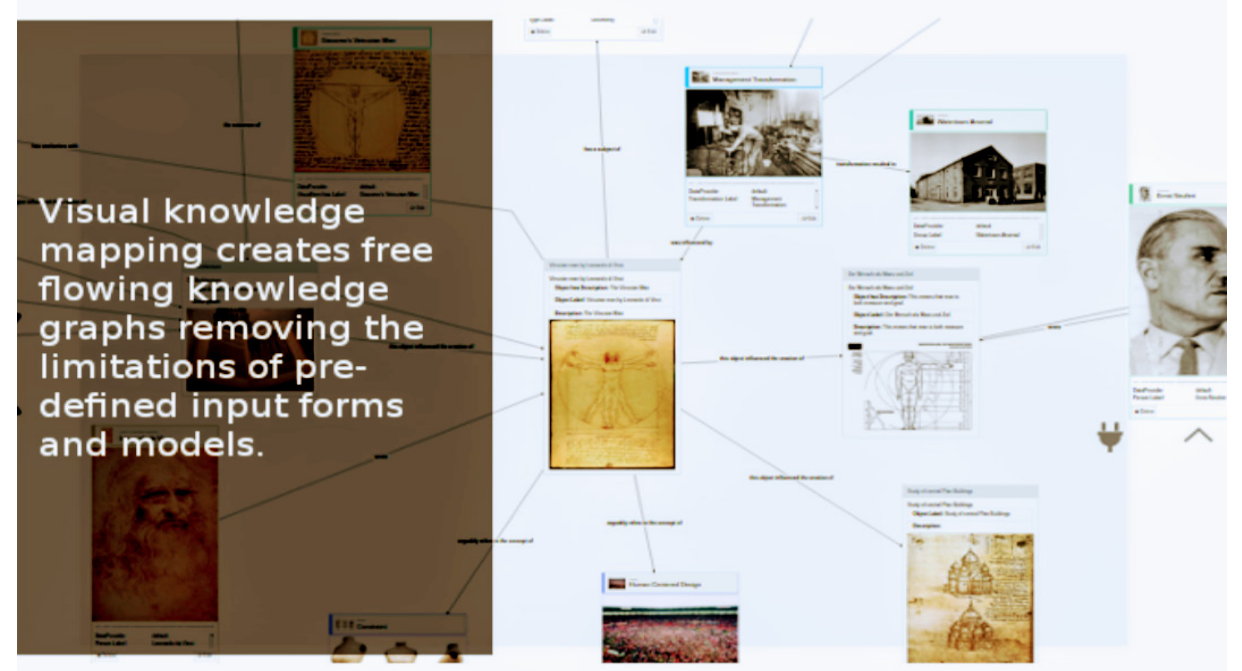

Fonte: www.public.researchspace.org

Os dados de diferentes fontes são integrados à base de dados Collection Online, através da aplicação da ontologia CIDOC-CRM. Esse Modelo Conceitual de Referência foi proposto pelo Comitê Internacional de Documentação do Conselho Internacional de Museus - ICOM - e tem como propósito estabelecer uma linguagem comum para sistemas de informação heterogêneos, permitindo sua integração. Por meio dele, informações sobre o patrimônio cultural podem ser recuperadas e trocadas, sendo que diferentes sistemas se tornam interoperáveis, ou seja, trocam dados com perdas mínimas 
de conteúdo e funcionalidade. Tem-se uma compreensão compartilhada de informações relacionadas ao patrimônio cultural, a partir de um quadro semântico comum e extensível (CARRASCO et al., 2015). Por meio dessa dinâmica integração de metadados baseada na ontologia CIDOC-CRM, 0 ambiente interativo ResearchSpace conecta informações sobre pessoas, lugares, períodos, objetos e conceitos, sem a perda de seu contexto original. Isso permite que a comunidade científica aprimore as informações disponíveis e estabeleça conexões entre os dados, em um processo colaborativo.

Novas aplicações tecnológicas podem ser desenvolvidas, customizando-se conteúdos e expandindo-se, exponencialmente, o alcance e 0 grau de utilização do acervo do Museu Britânico. As informações se estruturam em redes, abrangendo tanto abordagens lineares como não lineares sobre os conteúdos e encorajando diferentes perspectivas de análise. 0 ambiente é aberto, relacional, expansível e customizável. Ele está disponível na versão beta.

\section{Fluxograma 1 - Representação esquemática ResearchSpace}

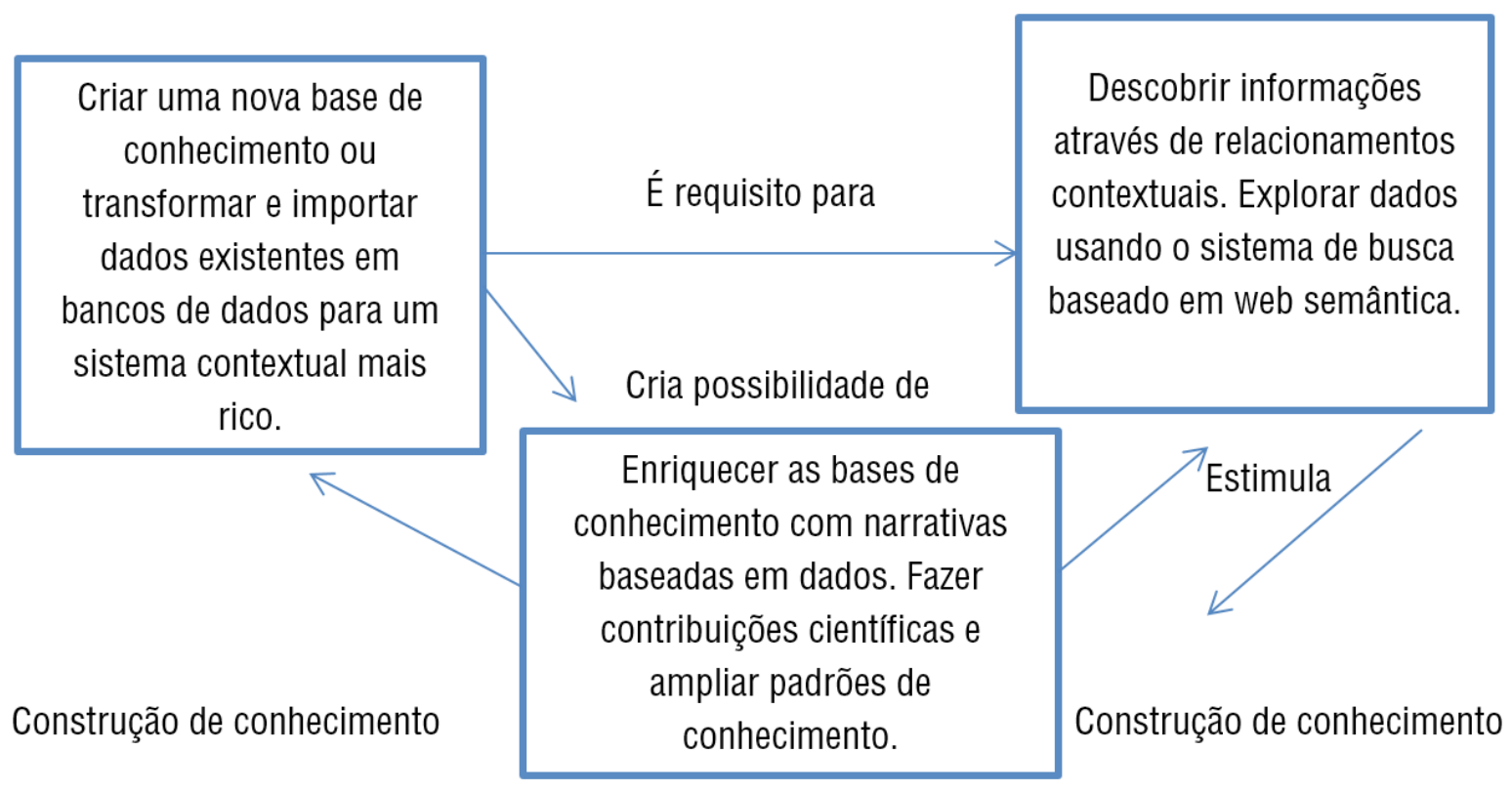

Fonte: adaptado de www.public.researchspace.org

0 projeto ResearchSpace propõe, assim, novos padrões de acessibilidade e uso do acervo digital do Museu Britânico. Ele permite incorporação de novos conjuntos de dados, trocas de informações com outras instituições culturais, interações com públicos-alvo, em tempo real, fomentando a geração de ideias e o desenvolvimento de projetos inovadores. Ele está em sintonia com o propósito do Museu 
Britânico de tornar o patrimônio cultural disponível, em escala global, para seus públicos-alvo. Observase que a gestão e análise de Big Data representam fontes de inovação para o Museu Britânico na medida em que criam as bases para que projetos informacionais como este sejam operacionalizados, estimulando o desenvolvimento de outros produtos e serviços, como desdobramento.

A plataforma virtual interativa "The Museum of the World - History Connected", desenvolvida pelo Museu Britânico em parceria com o Instituto Cultural Google, demonstra como a disseminação da cultura do Big Data nas organizações fomenta o desenvolvimento de aplicações tecnológicas inovadoras.

\section{Figura 5 - Plataforma virtual The Museum of the World}

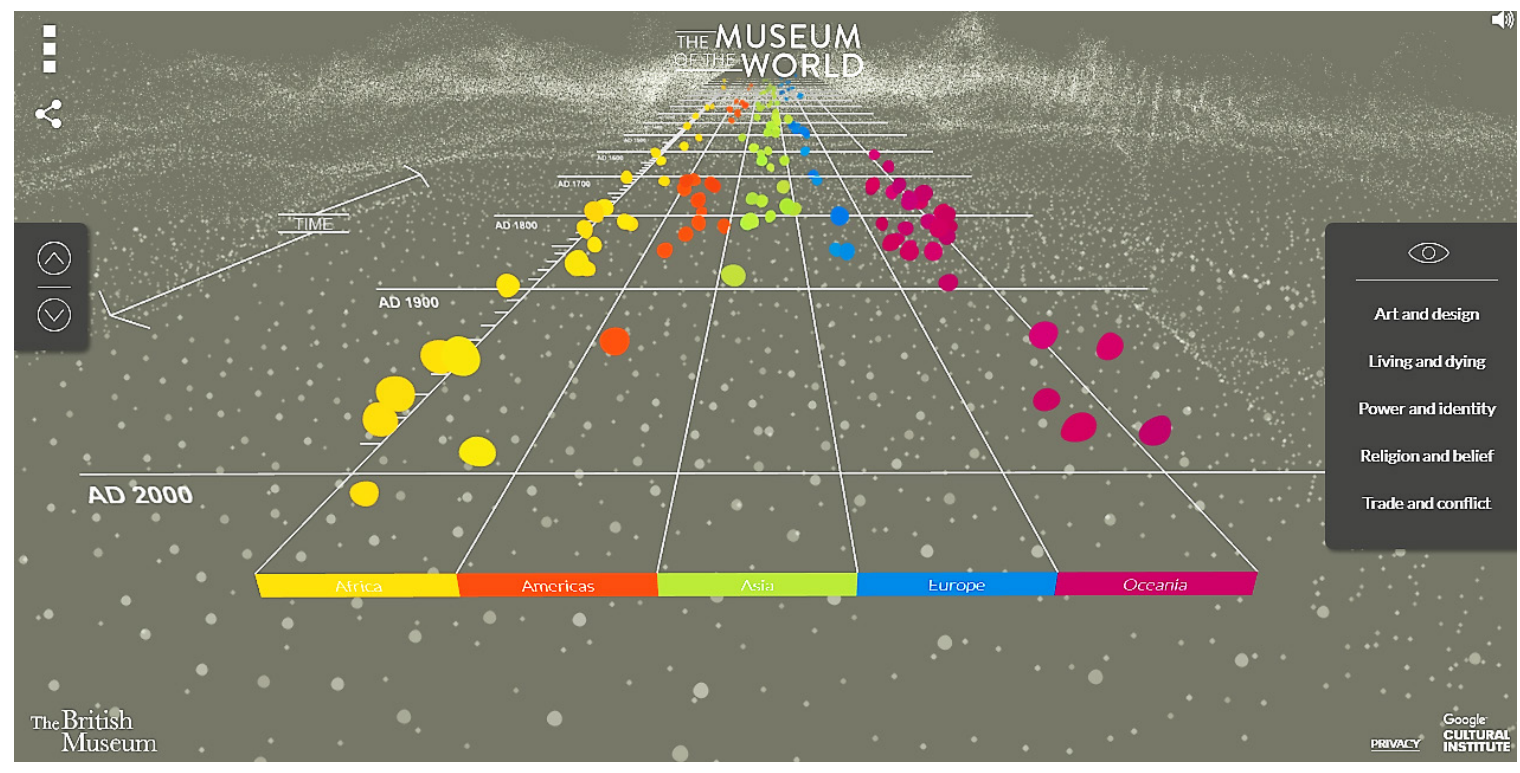

Fonte: www.britishmuseum.withgoogle.com

Esta plataforma virtual permite que 0 usuário explore parte da coleção de imagens digitais do acervo, associada a textos e áudio-descrições, navegando, por meio de uma linha do tempo, através de diferentes tempos, continentes e culturas. 0 Museu Britânico operacionalizou esse projeto através da tecnologia Web Graphics Library - WebGL, um Application Programming Interface - API - que possibilita a visualização bidimensional dos objetos museais e 0 estabelecimento de interconexões entre eles, a partir de diretrizes estabelecidas pelos curadores. Os objetos museais são relacionados entre si por meio de uma rede de hipertextos que estabelece conexões temáticas, dinamizada por cores, sons e animações. A plataforma cria a possibilidade dos usuários terem uma experiência única, diferenciada, a partir do acervo do Museu Britânico. 


\section{CONCLUSÃO}

Transformações digitais criam novos contextos e aplicações para as informações, em espaços museais físicos e virtuais, tornando-os cada vez mais inteligentes e interativos. Neles, passa a circular uma quantidade exponencial de dados, de diversas fontes e em diferentes formatos, que são gerenciados e transformados em informações estratégicas, por meio do Big Data. 0 processo permite que os museus aprimorem o uso de seus recursos, criem e compartilhem conhecimento, engajem públicos e aprimorem a experiência de museu destes, por meio de tecnologias avançadas.

A cultura do Big Data, disseminada nos museus, pode contribuir para que os processos de gestão se alinhem às diretrizes estratégicas destes. É possível observar, por meio do estudo de caso do Museu Britânico, que a gestão e análise de dados envolvem diversas etapas e são processos contínuos, guiados e retroalimentados por demandas gerenciais. Esses processos estimulam o desenvolvimento de projetos inovadores, baseados em estratégias de customização, de integração de tecnologias e em processos interativos, colaborativos.

\section{REFERÊNCIAS}

BAUTISTA, Susana Smith. Museums in the digital age. Changing Meanings of Place, Community and Culture. United Kingdom: AltaMiraPress, 2014.

BAX, Marcello Peixoto. A evolução da web rumo à web semântica. Revista Prisma.com. No. 19, 2012. Disponível em: http://ojs.letras.up.pt/index.php/prismacom/issue/view/153. Acesso em: 05/01/2019.

BRITISH MUSEUM, THE (website). Disponível em: www.britishmuseum.org. Acesso em: 20/11/2018.

CARRASCO, Laís; THALLER, Manfred; VIDOTTI, Silvana Aparecida Gregório Vidotti. Ontologia CIDOCCRM no contexto dos ambientes digitais de patrimônios culturais. Liinc em Revista. Rio de Janeiro, v. 11, n. 1, p. 208-222, 2015.

CHEN, C.L Philip; ZHANG, Chun-Yang. Data-intensive applications, challenges, techniques and Technologies: a survey on Big Data. Information Sciences. No. 275, p. 314-347, 2014. Disponível em: https://www.sciencedirect.com/science/article/pii/S0020025514000346. Acesso em: 05/01/2019.

$\mathrm{CHOO}$, Chun Wei. A organização do conhecimento: como as organizações usam a informação para criar significado, construir conhecimento e tomar decisões. São Paulo: Editora Senac, 2003.

CRESWELL, John W. Qualitative Inquiry \& Research Design. Choosing among five approaches. Los Angeles: Sage, 2013. 
DAISH, Alice. Transforming a museum to be data-driven using R. Anais da 12 $^{\text {a }}$. Conferência Internacional de Curadoria Digital - Upstream, Dowstream: embedding digital curation workflows for data science scholarship and society. 20 a 23 de fevereiro de 2017. Edimburgo. Disponível em: data-drive museums Zenodo (PDF).

DELOCHE, Bernard. El museo virtual: hacia una ética de las nuevas imágenes. Gijón: Ediciones Trea, 2001.

FRAG0SO, Suely; RECUER0, Raquel; AMARAL, Adriana. Métodos de pesquisa para internet. Porto Alegre: Sulina, 2013.

FREITAS, Wesley R.S.; JABBOUR, Charbel J.C. Utilizando estudo de caso(s) como estratégia de pesquisa qualitativa: boas práticas e sugestões. Revista Estudo \& Debate. Lajeado, v.18, n. 2, p.0722, 2011.

GANDOMI, Amir; HAIDER, Murtaza. Beyond the hype: Big Data concepts, methods and analytcs. International Journal of Information Management. No.35, p. 137-144, 2015. Disponível em: www. sciencedirect.com/science/article/pii/S0268401214001066. Acesso em: 05 jan. 2019.

GOBIRA, Pablo (org.). Percursos contemporâneos. Realidades da arte, ciência e tecnologia. Belo Horizonte: EdUEMG, 2018.

HAMMOUDI, Sarra; ALIOUAT, Zibouda; HAROUS, Saad. Challenges and research directions for Internet of Things. Telecommunication Systems. V. 67, n. 2, p.367-385, 2018.

JIN, Xiaolong; WAH, Benjamin W., CHENG, Xueq; WANG, Yuanzhuo. Significance and challenges of big data research. Big Data research 2. p. 59 -64, 2015.

KEENE, Suzanne. Digital collections: museums and the information age. Oxon: Routledge, 2011.

MARTINS, Tatiana Mara Alves. Crowdsourcing em museus: contribuições para a preservação do patrimônio na web 2.0. Dissertação de Mestrado. Escola de Ciência da Informação. Universidade Federal de Minas Gerais. 2017. Disponível em: http://www.bibliotecadigital.ufmg.br/dspace/ handle/1843/ECIP-B2LQY7 Acesso em: 05/01/2019.

MICROSOFT News Centre UK. The British museum is using big data to help visitors learn more about history (online). Disponível em: www.news.microsoft.com/en-gb/2017/07/04/the-britishmuseum-is-using-big-data-to-help-visitors-learn-more-about-history/. Acesso em: 10/11/2018.

MUSEUM OF THE WORLD, THE (website). Disponível em: https://britishmuseum. withgoogle.com/. Acesso em: 10 jan. 2019.

PATTON, Michael Quinn. Qualitative research and evaluation methods. Integrating theory and practice. Los Angeles: Sage, 2001.

PERERA, Kamani; CHANDRA, Dinesh. Knowledge management and museums. 23a . Conferência Anual CIDOC/ICOM - Knowledge management and museums. 04 a 09 de setembro de 2011, Romênia. Disponível em: http://network.icom.museum/fileadmin/user_upload/minisites/cidoc/ ConferencePapers/2011/PERERA_CHANDRA_CID0C_2011paper.pdf. Acesso em: 09/11/2018. 
RESEARCHSPACE (website). Versão Beta. Disponível em: www.researchspace.org. Acesso em: 05 jan. 2019.

SCHWAB, Klaus. The fourth industrial revolution. New York: Crown Business, 2016.

SIMON, Nina. The participatory museum. Santa Cruz: Museum 2.0, 2010.

TOWARDS 2020 - The British Museum Strategy (online). Disponível em:

www.britishmuseum.org/pdf/Towards_2020-The_British_Museum_Strategy.pdf. Acesso em: 15 dez. 2018.

WAMBA, Samuel Fosso; AKTER, Shahriar; EDWARDS, Andrew; CHOPIN, Geoffrey; GNANZOU, Denis. How big data can make big impact: Findings from a systematic review and a longitudinal case study. International Journal of Production Economics, v. 165, p. 234-246, 2015. 